\title{
Prevalência e determinantes de sintomatologia depressiva em idosos assistidos em serviço ambulatorial
}

\author{
Prevalence and determinants of depressive symptoms in elderly assisted in outpatient service
}

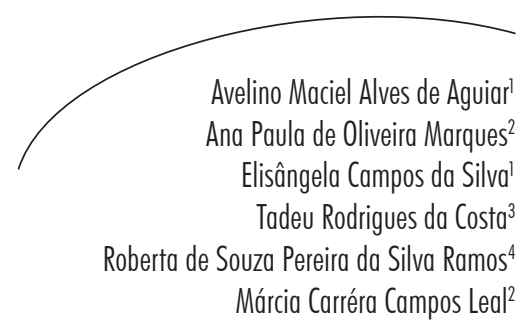

Resumo

Objetivo: Estimar a prevalência de sintomatologia depressiva e avaliar sua associação com fatores sociodemográficos e condições de saúde em idosos atendidos em serviço ambulatorial especializado geronto-geriátrico. Métodos: Estudo epidemiológico, descritivo, quantitativo, de corte transversal. A amostra foi composta por 301 idosos com 60 anos e mais, de ambos os sexos. Realizou-se entrevista face a face com cada participante, sendo o diagnóstico de sintomatologia depressiva definido pela utilização da Escala de Depressão Geriátrica em versão reduzida de Yesavage (GDS-15). Processaram-se os dados com programa SPSS 13.0, realizando-se estatística descritiva, teste de associações, utilizando o Qui-quadrado de Pearson, o Teste Exato de Fisher e o teste de comparação entre duas médias. Para verificação do efeito independente das variáveis, foi utilizado um modelo de regressão linear. Resultados: A prevalência global de sintomatologia depressiva correspondeu a 16,3\%, seguindo a tendência epidemiológica atual descrita na literatura. Quando analisadas através do modelo linear generalizado binomial, apenas autopercepção da saúde, comparação com a saúde e handicap auditivo se mostraram associadas. Conclusão: Estratégias voltadas à identificação dos fatores associados à depressão podem ajudar os diversos profissionais dos serviços de saúde, inseridos em qualquer nível de atenção, a compreender a realidade destes indivíduos, bem como diagnosticar e propor intervenções mais precoces e adequadas possíveis.

Programa de Pós-graduação Integrado em Saúde Coletiva, Departamento de Medicina Social. Universidade Federal de Pernambuco. Recife, PE, Brasil.

2 Programa de Pós-graduação Integrado em Saúde Coletiva e Programa de Pós-graduação em Enfermagem, Centro de Ciências da Saúde. Universidade Federal de Pernambuco da Universidade Federal de Pernambuco. Recife, PE, Brasil.

3 Programa de Pós-graduação em Biometria e Estatística Aplicada. Universidade Federal Rural de Pernambuco. Recife, PE, Brasil.

4 Programa de Pós-graduação em Enfermagem, Centro de Ciências da Saúde. Universidade Federal de Pernambuco. Recife, PE, Brasil.

Correspondência / Correspondence

Avelino Maciel Alves de Aguiar

E-mail: macielaguiar@hotmail.com

Palavras-chave:

Envelhecimento. Saúde

Mental. Depressão. Estudos

Transversais. 


\section{Abstract}

Objective: To estimate the prevalence of depressive symptoms and evaluate its association with sociodemographic factors and health status in elderly patients from specialized geronto-geriatric outpatient service. Methods: Epidemiological, descriptive, quantitative, cross-sectional study. The sample consisted of 301 elderly aged 60 and over, both sexes. Face to face interview was held with each participant, and the diagnosis of depressive symptoms were defined through the use of the Geriatric Depression Scale in reduced version of Yesavage (GDS-15). Data were processed with SPSS 13.0 software, performing descriptive statistics, associations test using the Chi-square test, Fisher's exact test and the test for comparison between two means. To check the effect of the independent variables, a linear regression model was used. Results: The overall prevalence of depressive symptoms accounted for $16.3 \%$, following the current epidemiological trend described in the literature. When analyzed using binomial generalized linear model, only self-perceived health, compared with health and hearing handicap were associated. Conclusion: Strategies aimed at identifying factors associated with depression may help many professional health services, entered at any level of attention, to understand the reality of these individuals, as well as diagnose and propose possible earlier and more appropriate intervention.
Key words: Aging. Mental Health. Depression. CrossSectional Studies.

\section{INTRODUÇÃo}

É fato inconteste que o Brasil, seguindo tendência mundial, passa por um processo de transição demográfica e epidemiológica, com aumento significativo da população idosa e ocorrência de doenças crônicas, múltiplas e onerosas. Elevada e crescente prevalência de transtornos mentais tem sido observada em idosos, ${ }^{1,2}$ e diante da complexidade dos fatores que determinam este agravo, estudos que investiguem as características sociodemográficas e de saúde desses indivíduos e sua associação com a depressão são importantes.

Segundo Drucker, ${ }^{1}$ a depressão é uma das doenças de maior interesse nos estudos relacionados ao envelhecimento, por suas consequências na qualidade de vida dos sujeitos. Traz repercussões altamente importantes para a vida do idoso, tanto sociais quanto individuais, pois afetam não somente o convívio social, impossibilitando uma rotina de vida satisfatória, mas também pelo risco inerente de morbidade e cronicidade. Provoca muitas vezes, no idoso, medo, insegurança e dificuldades no acesso aos recursos sociais e de saúde.

A depressão, além de repercutir em todos os aspectos de vida do idoso, gera, em termos de carga global, forte impacto para a saúde pública, por ser uma das principais causas de incapacidade, tendo como consequência a diminuição da capacidade funcional e da qualidade de vida, como também a alta taxa de utilização de serviços de saúde. Apresenta tendência ascendente nos próximos anos, e diversas investigações epidemiológicas demonstraram que a depressão impõe enorme sobrecarga econômica e social à sociedade. ${ }^{2}$

Considerando o exposto, este estudo teve como objetivo estimar a prevalência de depressão e sua associação com fatores sociodemográficos e condições de saúde em idosos atendidos em serviço ambulatorial geronto-geriátrico. Desta forma, espera-se que as informações contidas neste artigo possam colaborar na elaboração e aprimoramento das ações voltadas para a promoção da saúde e prevenção de agravos associados a este grupo etário. 


\section{MÉTODOS}

Tratou-se de estudo epidemiológico, descritivo, quantitativo, de corte transversal. A população do estudo foi de 1.834 idosos (60 anos e mais), de ambos os sexos, assistidos em um serviço gerontogeriátrico vinculado à Universidade Federal de Pernambuco (UFPE), que tiveram atendimento médico ou de enfermagem entre janeiro de 2006 e dezembro de 2010, de acordo com o registro dos prontuários existentes no serviço.

O tamanho da amostra foi calculado tendo por base estimativa uma prevalência de depressão nessa população de $50 \%$, por não haver consenso na literatura, em termos de magnitude do problema e em função das diferentes características da população estudada. Considerou-se um nível de confiança $95 \%$ e erro amostral de $5 \%$, perfazendo um total de 301 idosos.

Foi construído um banco de dados da população estudada no pacote Microsoft Excel e os idosos foram selecionados por meio de amostragem estratificada proporcional por sexo, com a seleção dos indivíduos realizada por sorteio sistemático, objetivando-se conseguir estimativas mais precisas e menor possibilidade de vieses de seleção. ${ }^{3}$ Os idosos sorteados foram convidados a participar da pesquisa por contato telefônico e as perdas foram repostas por novos sorteios que eram realizados quando necessário.

A coleta de dados foi feita através da aplicação de um roteiro de entrevista composto por questões fechadas. Foram excluídos os idosos que apresentaram enfermidades ou agravo à saúde em geral que pudessem comprometer o andamento e/ou a aplicação do roteiro de entrevistas, bem como a ida ao idoso ao local de coleta das informações.

A variável dependente foi a presença ou não de depressão, identificada pela Escala de Depressão Geriátrica - Abreviada de Yesavage (GDS-15), utilizando-se o ponto de corte em 5/6 (caso e não caso). ${ }^{4}$
As variáveis independentes foram divididas em dois blocos temáticos, sendo: a) sociodemográficas (sexo, faixa etária, situação conjugal, escolaridade em anos de estudo, arranjo familiar, contribuição para o sustento da casa, renda individual mensal e situação previdenciária); b) condições de saúde (autopercepção da saúde, comparação com a saúde, capacidade funcional, déficit cognitivo, morte importante no último ano, internações hospitalares nos últimos seis meses e handicap auditivo).

Quanto à autopercepção da saúde, o idoso(a) foi questionado em relação a sua saúde e em comparação com a saúde de outras pessoas da mesma idade. Foi distribuída nas categorias: excelente, muito boa, boa, regular e ruim, para a percepção de sua saúde, e nas categorias: muito pior, pior, melhor e muito melhor em relação à comparação de sua saúde. Foi dicotomizada pela combinação das categorias em "excelente", "muito boa" e "boa", para referir uma boa autopercepção de saúde; e em "regular" e "ruim", para referir uma autopercepção ruim. E "muito pior" e "pior" para referir uma comparação pior e melhor; e "muito melhor" para uma comparação melhor.

No que se refere à capacidade funcional, utilizou-se o Índice de Katz para avaliar as atividades básicas de vida diária (AVDs), ${ }^{5}$ onde 5 a 6 pontos corresponderam a independência; 3 a 4 pontos, a dependência moderada; e 2 pontos para dependência importante ou muito dependente. ${ }^{6}$ Para avaliar as atividades instrumentais de vida diária (AIVDs), utilizou-se a escala de Lawton. ${ }^{7}$ Para critérios de classificação, foram consideradas as pontuações: de 0 a 16 pontos como "dependência"; 17 e mais pontos, "independência"; e "máxima independência" em 27 pontos. $^{8}$

Na avaliação da função cognitiva, foi utilizado o Mini-Exame do Estado Mental (MEEM). Considerando a escolaridade, o ponto de corte, para indivíduos analfabetos foi $18 / 19$ e para aqueles com instrução escolar foi $24 / 25$. ? 
A desvantagem auditiva (handicap) "Relacionase aos aspectos não auditivos, resultantes da deficiência e da incapacidade auditivas, os quais limitam ou impedem o indivíduo de desempenhar adequadamente suas atividades de vida diária e comprometem suas relações na família, no trabalho e na sociedade." ${ }^{\prime 10}$ Sendo assim, o instrumento utilizado foi o questionário de autoavaliação The Hearing Handicap Inventory for the Elderly - Screening Version - HHIE-S, com adaptação para a língua portuguesa. ${ }^{11}$ É composto por dez questões, elaboradas para detectar problemas emocionais e sociais associados à perda auditiva.

Segundo Iorio \& Pinzan-Faria, ${ }^{12} \mathrm{O}$ questionário HHIE-S é um instrumento de triagem auditiva com alta especificidade e sensibilidade no rastreio de perdas auditivas em idosos que procuram serviços de saúde que não são específicos para atendimentos relacionados às alterações da audição.

Ainda dentro deste aspecto, foi questionado ao idoso se o mesmo apresentava dificuldades para escutar ou ouvir. Foi investigada, ainda, a percepção de sua saúde auditiva, distribuída nas categorias "excelente", "muito boa", "boa", "regular" e "ruim", dicotomizada pela combinação das categorias em "excelente", "muito boa" e "boa", para referir uma boa autopercepção de saúde auditiva, e em "regular" e "ruim", para referir uma autopercepção auditiva ruim.

O banco de dados com as informações da amostra foi obtido por meio de digitação dos cadastros dos idosos em dupla entrada, através do software SPSS versão 13.0.

Para alcançar os objetivos propostos, optouse por fazer uma análise com o intuito de avaliar as possíveis associações entre as variáveis. Além dessa abordagem, que é comumente encontrada na literatura, optou-se por avaliar os efeitos dessas variáveis por meio do modelo linear generalizado binomial ou também conhecido como logit, uma vez que a variável de interesse é uma dummy do tipo 0 ou 1.

Para a análise bivariada, as tabelas foram geradas a partir do software SPSS versão 13.0 e tratadas no pacote Microsoft Excel. Os testes de associação Qui-quadrado de Pearson e comparação de médias foram aplicados no SPSS versão 13.0, e o Teste Exato de Fisher foi realizado no software $\mathrm{R}$ versão 2.12.2. O nível de significância adotado para análise foi de 5\%.

Para o ajuste do modelo, foi utilizado o software $R$ versão 2.12.2. As variáveis explicativas foram escolhidas a partir da análise bivariada. Para compor o modelo, a variável deveria apresentar p-valor menor ou igual a 20\% no teste de associação. O nível de significância adotado nessa análise também foi de 5\%.

Após ajuste do modelo de regressão, foi necessário validá-lo por meio da análise de resíduos. Neste estudo tomaram-se três medidas para a validação do modelo final ajustado (Resíduo Componente do Desvio, Distância de Cook e gráfico de Quantis da Normal com Envelope).

O protocolo de pesquisa foi aprovado pelo Comitê de Ética e Pesquisa do Centro de Ciências Saúde da Universidade Federal de Pernambuco (CAAE no 0066.0.172.000-11). Os participantes assinaram ou realizaram impressão digital do Termo de Consentimento Livre e Esclarecido, no qual eram explicados os objetivos da pesquisa e garantida a confidencialidade das informações obtidas.

\section{RESULTADOS}

Quanto aos resultados da aplicação da GDS, para verificação da presença de sintomatologia depressiva, observou-se que o escore foi positivo para a presença de sintomatologia depressiva em cerca de $16,3 \%$.

De acordo com a tabela 1, pode-se traçar o seguinte perfil com relação à presença de sintomatologia depressiva quanto às variáveis sociodemográficas: foram idosos do sexo feminino, entre 70 e 79 anos, separados(as) ou divorciados(as), que estudaram entre um e quatro anos, que moravam com familiares, mas sem o companheiro, que não contribuíam para o sustento da casa, sem renda e que não eram aposentados. 
Analisando-se as associações dessas variáveis com a sintomatologia depressiva, observa-se ainda, na tabela 1, que as mesmas (sexo, faixa etária, situação conjugal, escolaridade, arranjo familiar, contribuição para o sustento da casa, renda individual mensal e situação previdenciária) não apresentaram associação com sintomatologia depressiva.

Tabela 1. Distribuição da sintomatologia depressiva segundo características sociodemográficas. NAI, Recife-PE, 2011.

\begin{tabular}{|c|c|c|c|c|c|c|}
\hline & \multicolumn{4}{|c|}{ GDS } & \multirow{3}{*}{ Total } & \multirow{3}{*}{$p$-valor } \\
\hline & \multicolumn{2}{|c|}{ Maior que 5} & \multicolumn{2}{|c|}{ Menor ou igual a 5} & & \\
\hline & $\mathrm{n}$ & $\%$ & $\mathrm{n}$ & $\%$ & & \\
\hline Sexo & & & & & & $0,718^{*}$ \\
\hline Masculino & 12 & 15,0 & 68 & 85,0 & 80 & \\
\hline Feminino & 37 & 16,7 & 184 & 83,3 & 221 & \\
\hline Total & 49 & 16,3 & 252 & 83,7 & 301 & \\
\hline Faixa etária & & & & & & $0,579 * *$ \\
\hline 60 a 69 anos & 26 & 16,0 & 136 & 83,9 & 162 & \\
\hline 70 a 79 anos & 21 & 18,1 & 95 & 81,9 & 116 & \\
\hline 80 ou mais & 2 & 8,7 & 21 & 91,3 & 23 & \\
\hline Total & 49 & 16,3 & 252 & 83,7 & 301 & \\
\hline Situação conjugal & & & & & & $0,671 * *$ \\
\hline Casado & 27 & 17,0 & 132 & 83,0 & 159 & \\
\hline Solteiro & 5 & 18,5 & 22 & 81,5 & 27 & \\
\hline Viúvo & 11 & 12,8 & 75 & 87,2 & 86 & \\
\hline Separado/Divorciado & 6 & 20,7 & 23 & 79,3 & 29 & \\
\hline Total & 49 & 16,3 & 252 & 83,7 & 301 & \\
\hline Escolaridade (em anos de estudo) & & & & & & $0,443 * *$ \\
\hline Nunca estudou/Analfabeto(a) & 5 & 17,2 & 24 & 82,8 & 29 & \\
\hline 1 a 4 anos & 20 & 20,0 & 80 & 80,0 & 100 & \\
\hline 5 a 8 anos & 5 & 8,6 & 53 & 91,4 & 58 & \\
\hline 9 a 11 anos & 12 & 16,9 & 59 & 83,1 & 71 & \\
\hline$\geq 12$ anos & 7 & 16,3 & 36 & 83,7 & 43 & \\
\hline Total & 49 & 16,3 & 252 & 83,7 & 301 & \\
\hline Arranjo familiar & & & & & & $0,641^{*}$ \\
\hline Sozinho(a) & 5 & 10,2 & 44 & 89,8 & 49 & \\
\hline Com companheiro(a) & 10 & 16,4 & 51 & 83,6 & 61 & \\
\hline Com companheiro(a) e familiares & 17 & 17,3 & 81 & 82,7 & 98 & \\
\hline Com familiares (sem o companheiro) & 17 & 18,3 & 76 & 81,7 & 93 & \\
\hline Total & 49 & 16,3 & 252 & 83,7 & 301 & \\
\hline
\end{tabular}


Contribuição para o sustento da casa

$0,431 * *$

\begin{tabular}{|c|c|c|c|c|c|c|}
\hline Sim, totalmente & 24 & 16,9 & 118 & 83,1 & 142 & \\
\hline Sim, parcialmente & 18 & 14,0 & 111 & 86,0 & 129 & \\
\hline Não contribui & 7 & 23,3 & 23 & 76,7 & 30 & \\
\hline Total & 49 & 16,3 & 252 & 83,7 & 301 & \\
\hline enda individual mensal & & & & & & $0,243^{* *}$ \\
\hline < 1 salário mínimo (Até $\mathrm{R} \$ 544,00$ ) & 7 & 23,3 & 23 & 76,7 & 30 & \\
\hline 1 a 2 salários $(\mathrm{R} \$ 545,00$ a $1.090,00)$ & 25 & 15,0 & 142 & 85,0 & 167 & \\
\hline$>2$ a 4 salários $(\mathrm{R} \$ 1.091,00$ a 2.180,00) & 7 & 13,0 & 47 & 87,0 & 54 & \\
\hline$>4$ salários ( $\mathrm{R} \$ 2.181,00$ ou mais) & 3 & 11,1 & 24 & 88,9 & 27 & \\
\hline Sem renda & 7 & 30,4 & 16 & 69,6 & 23 & \\
\hline Total & 49 & 16,3 & 252 & 83,7 & 301 & \\
\hline tuação previdenciária & & & & & & $0,715^{* *}$ \\
\hline Aposentado & 30 & 15,8 & 160 & 84,2 & 190 & \\
\hline Pensionista & 7 & 14,6 & 41 & 85,4 & 48 & \\
\hline Aposentado + Pensionista & 3 & 13,0 & 20 & 87,0 & 23 & \\
\hline Não é aposentado & 9 & 22,5 & 31 & 77,5 & 40 & \\
\hline Total & 49 & 16,3 & 252 & 83,7 & 301 & \\
\hline
\end{tabular}

* Teste Qui-quadrado; ** Teste Exato de Fisher.

Traçando o perfil segundo as condições de saúde, tem-se que os idosos que apresentam maiores proporções de GDS, isto é, pontuação maior que 5 como indicativo de sintomatologia depressiva, são aqueles que: autoavaliaram sua saúde como ruim; que consideram sua saúde pior do que a de outros idosos; que são muito dependentes segundo o índice de Katz; que apresentam dependência segundo a escala de Lawton; que apresentam déficit cognitivo ou não; que perderam alguém importante no último ano; e que passaram por internações hospitalares nos últimos seis meses (tabela 2 ).

Ainda nesse aspecto, quanto às variáveis relacionadas à saúde, na tabela 2 é possível observar também que a autopercepção da saúde apresentou associação com a sintomatologia depressiva de tal forma que, ao declarar uma boa saúde, tem-se um percentual muito baixo de idosos com GDS maior que 5; e ao declarar sua saúde ruim, o percentual de idoso com GDS maior que 5 aumenta de 2,0\% para 23,6\%.
Com relação à comparação de sua saúde com a de outros idosos, o Teste Exato de Fisher indicou haver associação desta variável com a sintomatologia. Analisando tal associação, observa-se que os idosos que apresentam GDS maior que 5 declaram que sua saúde é pior do que a de outros idosos. Já com os idosos que apresentam GDS menor ou igual que 5, a grande maioria declara que sua saúde é melhor do que a de outros idosos.

O Índice de Katz e a Escala de Lawton também apresentaram associação com a GDS. Quanto ao Índice de Katz, $100 \%$ dos que apresentaram muita dependência, tiveram um escore para a GDS maior que 5. Para dependência moderada, a distribuição de idosos na GDS foi balanceada: 50\% de idosos para cada categoria da mesma. Quanto aos que foram classificados como independentes, a maioria $(85,5 \%)$ apresentou GDS menor ou igual a 5. A escala de Lawton apresentou distribuição similar ao Índice de Katz. Para a classificação 
"independência", a maioria $(84,8 \%)$ dos idosos apresentou GDS menor ou igual a 5. Para a classificação "dependência", a quantidade de idosos com GDS maior ou igual a 5 foi maior, mesmo que com uma diferença mínima.
Foi observado também que déficit cognitivo (MEEM), morte importante no último ano e internações hospitalares nos últimos seis meses não apresentaram associação com sintomatologia depressiva, como descrito na tabela 2.

Tabela 2. Distribuição da sintomatologia depressiva segundo características de condição de saúde. NAI, Recife-PE, 2011.



* Teste Qui-quadrado; ** Teste Exato de Fisher. 
Com relação ao perfil da GDS segundo as variáveis associadas à autoavaliação da audição, tem-se que aqueles que apresentam dificuldades para ouvir/escutar e que autoavaliam sua audição como ruim apresentam sintomatologia depressiva, a partir dos dados apresentados na tabela 3.

Em termos de handicap auditivo, ainda na tabela 3, observa-se que as médias do mesmo para GDS menor ou igual a 5 e maior que 5 diferem segundo o teste de comparação de médias. Além disso, tal diferença é quase o dobro para a pontuação da GDS maior que 5, que apresenta média de handicap de 13,3, indicando que os idosos com GDS maior que 5 apresentam valores médios de bandicap maiores que os idosos que possuem GDS menor ou igual a 5 - ou seja, observa-se associação entre handicap auditivo e sintomatologia depressiva.

Foi verificado também que as variáveis "dificuldades para ouvir/escutar" e "autoavaliação da audição" apresentaram associações com sintomatologia depressiva. Quanto à primeira variável, verifica-se que o percentual de idosos com GDS maior que 5 aumenta quando os idosos apresentam dificuldades para ouvir/ escutar. Com relação à autoavaliação da audição, ao avaliarem sua audição como ruim, tem-se um acréscimo de idosos com GDS maior que 5, indicando sintomatologia depressiva.

Tabela 3. Distribuição da sintomatologia depressiva segundo características do handicap auditivo e de autoavaliação da audição. NAI, Recife-PE, 2011.

\begin{tabular}{|c|c|c|c|c|c|c|}
\hline & \multicolumn{4}{|c|}{ GDS } & \multirow{2}{*}{ Total } & \multirow{2}{*}{$p$-valor } \\
\hline & \multicolumn{2}{|c|}{ Maior que 5} & \multicolumn{2}{|c|}{ Menor ou igual a 5} & & \\
\hline Handicap & & & & & & $<0,001^{* *}$ \\
\hline Média & \multicolumn{2}{|c|}{13,3} & \multicolumn{2}{|c|}{6,7} & 7,8 & \\
\hline Desvio-padrão & \multicolumn{2}{|c|}{10,3} & \multicolumn{2}{|c|}{7,5} & 8,4 & \\
\hline Mínimo & \multicolumn{2}{|c|}{0} & \multicolumn{2}{|c|}{0} & 0,0 & \\
\hline \multirow[t]{2}{*}{ Máximo } & \multicolumn{2}{|c|}{40} & \multicolumn{2}{|c|}{36} & 40,0 & \\
\hline & $\mathrm{n}$ & $\%$ & $\mathrm{n}$ & $\%$ & Total & $p$-valor \\
\hline Dificuldades para ouvir/ escutar & & & & & & $0,004^{*}$ \\
\hline Não & 19 & 10,9 & 156 & 89,1 & 175 & \\
\hline $\operatorname{Sim}$ & 30 & 23,8 & 96 & 76,2 & 126 & \\
\hline Total & 49 & 16,3 & 252 & 83,7 & 301 & \\
\hline Autoavaliação da audição & & & & & & $0,031^{*}$ \\
\hline Boa & 20 & 12,1 & 145 & 87,9 & 165 & \\
\hline Ruim & 29 & 21,3 & 107 & 78,7 & 136 & \\
\hline Total & 49 & 16,3 & 252 & 83,7 & 301 & \\
\hline
\end{tabular}

* Teste Qui-quadrado; ** Teste de comparação entre duas médias. 
No que tange ao ajuste do modelo, após aplicar o método stepwise tomando como referência as variáveis que apresentaram p-valor menor ou igual que $20 \%$ na análise bivariada, como pode ser verificado na tabela 4 , o modelo final é dado por:

$\hat{\eta}_{i}=-4,29+2,35 \mathrm{X}_{1 i}-1,69 \mathrm{X}_{2 i}+0,06 \mathrm{X}_{8 i}$

Analisando as razões de chance, quando o idoso autoavalia sua saúde como ruim, a chance de apresentar quadros depressivos aumenta em $945 \%$. Com relação à comparação de sua saúde com a de outros idosos, quando o idoso afirma que sua saúde é pior, a chance de apresentar sintomatologia depressiva aumenta em 443\%. Para a desvantagem auditiva, quando o idoso aumenta em uma unidade seu handicap, temse uma chance de $6 \%$ de apresentar sintomas depressivos.

Tabela 4. Resultados do ajuste do modelo final após aplicação do método stepwise. NAI, Recife-PE, 2011.

\begin{tabular}{lcccc}
\hline & Estimativa & Erro padrão & $p$-valor & $\begin{array}{c}\text { Razão de } \\
\text { chances }\end{array}$ \\
Constante & $-4,29$ & 0,74 & $<0,001$ & 0,01 \\
Autoavaliação da saúde & 2,35 & 0,74 & 0,002 & 10,45 \\
Comparação com a saúde de outros idosos & 1,69 & 0,57 & 0,003 & 5,43 \\
Handicap & 0,06 & 0,02 & 0,003 & 1,06 \\
\hline
\end{tabular}

$\mathrm{R}^{2}$ de Nagelkerke $=0,28$

Analisando os resíduos a fim de validar o modelo final ajustado, segundo a figura 1(a), os resíduos apresentam bom comportamento aleatório, concentrando-se entre os valores 0 e -1 , com poucas observações se distanciando dos limites.

Na figura 1(b), observa-se que a maioria das observações se encontra próxima do ponto zero, com poucas observações sendo consideradas possivelmente influentes por se afastarem fortemente.

O gráfico de quantis da Normal com envelope, na figura 1 (c), indica que a suposição de que os erros são binomiais foi validada, uma vez que nenhum resíduo estimado ultrapassou o envelope. Além disso, observa-se que o modelo conseguiu captar o comportamento estudado.

Com relação aos pontos que se distanciaram dos demais na figura $1(\mathrm{~b})$, referente ao gráfico da distância de Cook, estes foram retirados e o ajuste e os resíduos foram reavaliados. Como os pontos foram retirados fazendo todas as combinações possíveis entre eles, a quantidade de modelos ajustados e de análise de resíduo realizada foi muito alta, e como não é foco entrar nos detalhes, apresenta-se o que foi encontrado a seguir de forma textualizada e resumida.

A retirada dos pontos não melhorou o ajuste. Pelo contrário, manteve o ajuste até certo ponto constante, mas ao realizar novamente a análise de resíduos para cada grupo de pontos retirados, observou-se maior espalhamento dos pontos no gráfico da distância de Cook e leves alterações no gráfico de quantis da Normal.

Com base nos resultados obtidos na análise de resíduos, verifica-se que o modelo final ajustado é bem ajustado com relação às suposições básicas, mas que apresenta baixo nível de explicação (28\% segundo o método de Nagelkerke). Mesmo assim, o modelo final ajustado foi considerado o melhor, levando em consideração a amostra utilizada neste estudo. 


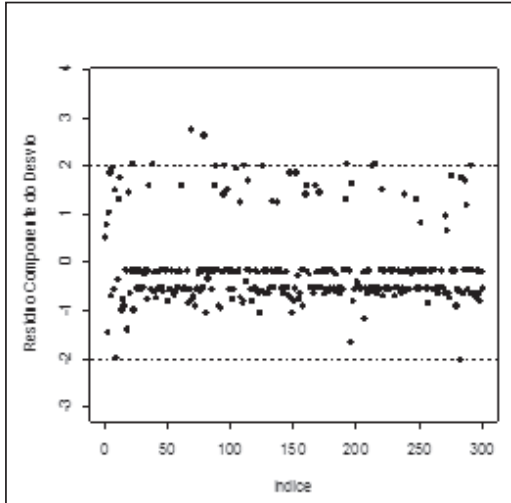

(a)

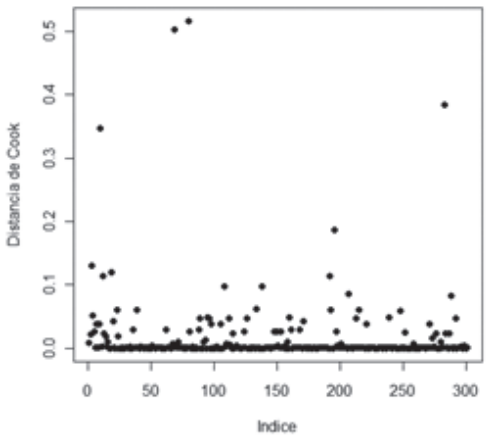

(b)

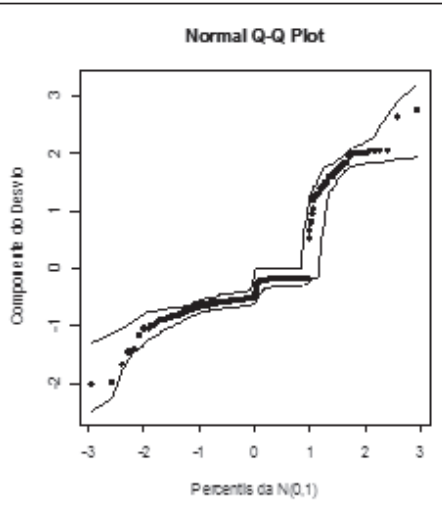

(c)

Figura 1. Resíduo Componente do Desvio do modelo final (a); Distância de Cook (b); Gráfico de quantis da Normal para distribuição Binomial com envelope (c).

\section{DISCUSSÃO}

Quanto à prevalência de sintomatologia depressiva, as comparações do presente estudo com outros anteriores requerem alguns cuidados, uma vez que a frequência de depressão nessa população dependerá do contexto no qual está inserida, procedimentos metodológicos utilizados e principalmente no que se refere ao tipo de instrumento utilizado para sua mensuração. ${ }^{13}$ Desta forma, a prevalência de depressão no idoso tem sido alvo de discussões em virtude de resultados díspares nos estudos. ${ }^{14,15}$

Neste estudo, a prevalência de quadros depressivos, entre os 301 idosos que compuseram a amostra, foi estimada em 16,3\%, considerando o ponto de corte 5/6 para caso/não caso. Buscando confrontar tal achado com estudos que utilizaram a GDS-15, observou-se que menor prevalência foi encontrada em pesquisa epidemiológica no município de Botucatu-SP $(15,6 \%) .^{8}$ Valores próximos aos encontrados neste estudo encontram-se em estudo realizado em Florianópolis-SC $(17,4 \%)^{16}$ e no município de São Paulo-SP $(18,1 \%) \cdot{ }^{17}$

No entanto, pesquisas têm demonstrado prevalências superiores à encontrada. . $^{1518-22}$ Destaca-se ainda que, embora empregando o mesmo instrumento, algumas destas pesquisas utilizaram pontos de corte distintos no diagnóstico de sintomatologia depressiva.

Quanto às variáveis sociodemográficas e sua associação com a sintomatologia depressiva, no que concerne à variável "sexo", a pesquisa aponta que as mulheres apresentaram maior frequência (16,7\%), fato comprovado por diversas publicações nacionais e internacionais no que se refere à epidemiologia da depressão. . $^{1518,19,21,23-25}$

Podem-se destacar também os fatores socioculturais associados a experiências adversas e fatores psicológicos associados com maior vulnerabilidade a eventos estressores, que também contribuem para tal diferença. ${ }^{23,26,27}$ Além disso, as mulheres têm maior probabilidade de admitir, ou verbalizar seus sentimentos que os homens; estes, na maioria das vezes, tendem a negar seus sentimentos ou utilizar recursos como alcoolismo e tentativas de suicídio. ${ }^{14,28}$

Quanto à variável "idade”, observou-se maior proporção de idosos com idades entre 70 e 79 anos $(18,1 \%)$, corroborando o que foi apontado em estudo sobre depressão e envelhecimento na cidade de Recife-PE. ${ }^{14}$ Entretanto, não existe consenso bem documentado na literatura mundial sobre a faixa etária de maior prevalência dessa doença. ${ }^{20,29}$ Estudos epidemiológicos sugerem que os efeitos da idade, na sintomatologia 
depressiva, podem ser atribuídos aos problemas de saúde e incapacidades frequentes, e não ao envelhecimento per $s i_{.}^{29,30}$

Verificou-se também, quanto à situação conjugal, prevalência de depressão provável superior nos idosos separados/divorciados $(20,7 \%)$. Não obstante, verifica-se quase que uma uniformidade entre as proporções de sintomatologia depressiva nos estratos (casado, solteiro, viúvo e separado/divorciado), destacando-se que a menor frequência esteve descrita entre os viúvos (12,8\%). Este resultado vem em oposição a outros estudos, os quais apontam que a solidão ou luto pela morte de um cônjuge na senilidade pode ser fator de risco para desenvolvimento de sintomas depressivos. ${ }^{15,21}$

Outro aspecto a se destacar, quanto ao arranjo familiar dos idosos investigados, foi que este apresentou linearidade quanto à frequência de sintomatologia depressiva, e seu maior percentual foi apontado naqueles que vivem com familiares, mas sem a presença do companheiro(a), totalizando $18,3 \%$ dos casos, apontando para a presença de lares multigeracionais.

Quanto à escolaridade, observou-se que a maior frequência de sintomas depressivos foi descrita entre idosos com até quatro anos de estudo $(20,0 \%)$ ou os que nunca estudaram ou são analfabetos $(17,2 \%)$, fato descrito na literatura. ${ }^{14,23,24}$

Pode-se observar, também, que a maior frequência de sintomatologia esteve presente nos idosos que não possuíam renda $(30,4 \%)$ ou nos que possuíam renda inferior a um salário mínimo (23,3\%), nos que não eram aposentados $(22,5 \%)$ e que não contribuíam para o sustento da casa $(23,3 \%)$. Em relação ao último tópico, se tomar como base contribuir ou não para o sustento da casa, sem levar em conta os critérios "totalmente" ou "parcialmente", observase que a maior frequência esteve para os que contribuíam através do somatório "totalmente e parcialmente" (30,9\%).

Esses dados inferem que o nível econômico apresenta relação inversa com os sintomas depressivos, de maneira que indivíduos mais desfavorecidos economicamente apresentam maiores índices de depressão, resultado presente em outros estudos populacionais. ${ }^{15,23,31}$

Finalizando o bloco das variáveis sociodemográficas, apesar de se observar diferenças nas proporções dos estratos analisados, não foi possível inferir associações estatísticas significativas das variáveis na análise bivariada. Ou seja, não foram obtidos resultados significativos ( $\mathrm{p}$-valor $<0,05)$ quanto a: sexo, faixa etária, situação conjugal, escolaridade, arranjo familiar, contribuição para o sustento da casa, renda e situação previdenciária dos idosos investigados.

Esses achados, apesar de irem de encontro a algumas pesquisas, nas quais se verificam associações dessas variáveis com a sintomatologia depressiva, corroboram outros estudos que também não verificaram associações em relação ao sexo, ${ }^{14,15}$ faixa etária, ${ }^{14,15,22,25}$ situação conjugal, ${ }^{14,15,22,25}$ escolaridade, ${ }^{15,22,25}$ arranjo familiar, ${ }^{15}$ renda $^{14,22}$ e situação previdenciária. ${ }^{22}$

No tocante à variável "autopercepção da saúde", dentro do conjunto das condições de saúde foi possível observar que a maioria dos idosos investigados referia ter uma saúde ruim (52,5\%), como também afirmaram que sua saúde se apresentava pior $(79,7 \%)$ quando comparada a de outros idosos.

Além disso, foi possível verificar forte associação com a sintomatologia depressiva na análise bivariada, tanto para a autopercepção da saúde ( $\mathrm{p}$-valor $<0,001$ ), quanto para a comparação com a saúde ( $p$-valor $<0,001)$. Esse fato é bastante notório na literatura gerontológica nacional e internacional, onde a autopercepção de saúde, além de importante indicador de bem-estar individual e coletivo, apresenta-se como robusto preditor de depressão na população idosa, pois se mostra inteiramente afetada pela presença de sintomas depressivos. ${ }^{18,31}$

Outro aspecto a se considerar seria a capacidade funcional na população idosa, em que esta se apresenta como um dos grandes 
indicadores de saúde nessa população em termos da habilidade e independência para realizar determinadas atividades. Desta forma, quando analisadas as AVDs e AIVDs com a sintomatologia depressiva, pode-se observar, no presente estudo, forte associação para ambas na análise bivariada ( $\mathrm{p}$-valor $<0,001$ e p-valor $=0,02$ ). Verificou-se, ainda, que há certa tendência na diminuição da independência destas quando se passa de não caso para caso.

Esses dados corroboram o estudo realizado na cidade de Botucatu-SP ${ }^{22}$ e pesquisa realizada na cidade de Natal-RN, ${ }^{18}$ além de estudo no município de Fortaleza-CE, ${ }^{15}$ nos quais foi possível constatar que de oito idosos que apresentavam algum comprometimento para AVDs, seis estavam entre os casos de depressão segundo a Escala de Depressão Geriátrica, demonstrando a correlação, nessa população, entre algum grau de dependência no dia a dia e sintomas depressivos. ${ }^{15}$

Dando seguimento, pode-se verificar que na associação entre comprometimento cognitivo e estado depressivo, não houve diferenças significativas nas frequências entre os dois estratos (sem e com déficit cognitivo), bem como na associação bivariada ( $\mathrm{p}$-valor $>0,99$ ). Embora esses dados possam ser confirmados por outros estudos, ${ }^{32}$ pesquisas demonstram que déficits cognitivos, avaliados a partir do MEEM, estão associados com o aparecimento de depressão. ${ }^{16}$

No que concerne à presença de morte significativa no último ano, observou-se maior proporção de idosos que afirmaram ter existido (17,2\%). Não foi possível, no entanto, estabelecer associações na análise bivariada ( $\mathrm{p}$-valor $=0,769)$, contrapondo-se ao estudo realizado na cidade de Pelotas-RS. ${ }^{23}$

Quanto a internações hospitalares nos últimos seis meses, constatou-se maior frequência de idosos com sintomatologia depressiva que relataram ter sido internados no último semestre $(33,3 \%)$. Apesar disso, não foi possível constituir associação desta variável na análise bivariada $(p$-valor $=0,09)$. Esses dados confirmam o estudo realizado no município de Fortaleza-CE, ${ }^{15}$ que verificou a autoavaliação da audição dos idosos e as dimensões subjetivas da perda auditiva que não aparecem no audiograma, ou seja, as consequências emocionais, sociais e de situação percebidas em função de um possível déficit auditivo e sua relação com sintomas depressivos. Mesmo considerando que o exame audiométrico seja padrão na detecção e quantificação de perdas auditivas, este se apresenta como um exame objetivo e não é suficiente para demonstrar a real habilidade ou dificuldade comunicativa e de desempenho psicossocial do sujeito avaliado diante de um possível déficit auditivo. ${ }^{33}$

De acordo com o resultado apresentado, observou-se que os idosos com sintomatologia depressiva apresentaram valores médios de desvantagem auditiva superiores aos não casos (quase o dobro quando comparados casos e não casos), maior frequência em relatar dificuldades auditivas $(23,8 \%)$ e maior proporção em autoavaliarem sua saúde auditiva como ruim (onde essa foi dicotomizada para a análise de associações). Foi possível também verificar associações significativas dessas três variáveis com sintomas depressivos no modelo de análise bivariada ( $\mathrm{p}$-valor $<0,001, \quad \mathrm{p}$-valor $=0,004 \mathrm{e}$ $\mathrm{p}$-valor $=0,031$, respectivamente).

Os dados apresentados indicaram interrelação entre desvantagem auditiva/déficit auditivo e sintomatologia depressiva. Segundo a literatura científica, a deficiência auditiva no idoso pode originar ou agravar quadros depressivos, isto é, quando a comunicação é prejudicada, pode ocorrer frustração diante da situação de interlocução, levando o indivíduo ao isolamento social e à depressão. ${ }^{10}$

Quanto ao modelo linear generalizado binomial, após ajuste, pode-se observar que apenas as variáveis "autopercepção da saúde", "comparação com a saúde" e "desvantagem auditiva" se mostraram associadas à sintomatologia depressiva ( $p$-valor $<0,05)$. Isso aponta que $o$ mesmo foi sensível para determinar quais variáveis da análise bivariada permaneceriam associadas à sintomatologia depressiva. 
Apesar de esse modelo ter um poder de explicação de apenas $28 \%$, através da análise de resíduo foi possível identificar sua validade, garantindo as comparações realizadas. Sugerese, assim, que o uso desse modelo possa ser de extrema importância para estudar os efeitos dessas e de outras variáveis frente à sintomatologia depressiva na população idosa, sendo necessário maior aprofundamento na sua modelagem metodológica. Em toda a revisão de literatura para compor o presente artigo, não foi identificado o uso desse tipo de modelagem nos estudos realizados.

No que concerne às limitações deste estudo, pode-se destacar: o uso de roteiro estruturado sujeito a distorções e tendenciosidade do entrevistador e/ou do entrevistado (mesmo que este viés possa ter sido minimizado pela realização do teste piloto) e o delineamento transversal, que apesar de apresentar vantagens como a rapidez e o baixo custo - requer algumas precauções na interpretação das associações, em função das dificuldades em estabelecer a relação temporal entre o desfecho e exposições.

\section{REFERÊNCIAS}

1. Drucker C. Religiosidade, crenças e atitudes em idosos deprimidos: em um serviço de saúde mental de São Paulo [dissertação]. São Paulo: Universidade Estadual de Campinas; 2005.

2. Nardi EFR, Andrade OG. Estados depressivos entre idosos na comunidade Jandaia do Sul, Paraná, Brasil. Arq Ciências Saúde UNIPAR 2005;9(2):109-11.

3. Jekel JF, Katz DL, Elmore JG. Epidemiologia, bioestatística e medicina preventiva. $2^{\mathrm{a}}$ ed. São Paulo: Artmed; 2005.

4. Paradela EMP, Lourenço, RA, Veras RP. Validação da escala de depressão geriátrica em um ambulatório geral. Rev Saúde Pública 2005;39(6):918-23.

5. Katz S, Downs TD, Cash HR, Grotz RC. Progress in development of the index of ADL. Gerontologist 1970;10(1):20-30.

6. Duarte YA, Andrade CL, Lebrão ML. O Índex de Katz na avaliação da funcionalidade dos idosos. Rev Esc Enferm USP 2007;41(2):317-25.
Para minimizar o problema acima referido, evitou-se inferir relações causais entre essas variáveis e o desfecho. Destaca-se também que não se pode desconsiderar, entretanto, a possibilidade de causalidade reversa nas associações encontradas.

\section{CONCLUSÃO}

De modo geral, os resultados deste estudo estão condizentes com os dados encontrados na literatura nacional e internacional, que apontam prevalências crescentes e significativas de depressão em idosos e sua associação com fatores sociodemográficos e condição de saúde.

Diante desse cenário, estratégias voltadas à identificação dos fatores associados à depressão podem ajudar os diversos profissionais dos serviços de saúde, em equipes multidisciplinares/ interdisciplinares, inseridos em qualquer nível de atenção (primária, secundária ou terciária), a compreender a realidade desses indivíduos, diagnosticar e propor intervenções mais precoces e adequadas.
7. Lawton MP, Brody EM. Assessment of older people: self-maintaining and instrumental activities of daily living. Gerontologist 1969;(9):179-86.

8. Netto MC. Validade e coerência de instrumentos utilizados em avaliações clínicas de idosos [dissertação]. São Paulo: Universidade Estadual Paulista "Júlio de Mesquita Filho”; 2008.

9. Veras RP, Lourenço RA. Mine-exame do estado mental: características psicométricas em idosos ambulatoriais. Rev Saúde Pública, 2006; 40(4):712-19.

10. Russo ICP. Intervenção Fonoaudiológica na Terceira Idade. Rio de Janeiro: Revinter; 2004. Distúrbios da audição: a presbiacusia; p. 51-82.

11. Wieselberg MB. A auto-avaliação do handicap em idosos portadores de deficiência auditiva: o uso do HHIE [dissertação]. São Paulo: Pontifícia Universidade Católica de São Paulo; 1997.

12. Iorio CM, Pinzan-Faria VM. Sensibilidade auditiva e autopercepção do handicap: um estudo em idosos. Distúrb Comun 2004;16(3):289-99. 
13. Crepaldi AL. Depressão e sintomas depressivos em idosos de baixa renda em São Paulo: prevalência, fatores associados e uso de serviços de saúde [Dissertação]. São Paulo: Universidade de São Paulo, Faculdade de Medicina; 2009.

14. Leite VMM, De Carvalho EMF, Barreto KML, Falcão IV. Depressão e envelhecimento: estudo nos participantes do Programa Universidade Aberta à Terceira Idade. Rev Bras Saúde Matern Infant 2006;6(1):31-8

15. Bandeira CB. Perfil dos idosos com depressão em comunidade do município de Fortaleza. Rev Bras Med Fam Comunidade 2008;4(15):189-204.

16. Borges LJ, Benedetti TRB, Mazo GZ. Rastreamento cognitivo e sintomas depressivos em idosos iniciantes em programa de exercício físico. J Bras Psiquiatr 2007;56(4):273-9.

17. Lebrão ML, Laurenti R. Saúde, bem-estar e envelhecimento: o estudo SABE no município de São Paulo. Rev Bras Epidemiol 2005;8(2):127-41.

18. Maciel ACC, Guerra RO. Influência dos fatores biopsicossociais sobre a capacidade funcional de idosos residentes no Nordestes do Brasil. Rev Bras Epidemiol 2007;10(2):178-89.

19. Ferrari JF, Dalacorte RR. Uso da Escala de Depressão Geriátrica de Yesavage para avaliar a prevalência de depressão em idosos hospitalizados. Sci Med 2007;17(1):3-8.

20. De Souza ALF, Fonseca DMC, De Almeida GSC, Gomes OE, Polese JC, Da Silva SL, et al. Frequência de suspeita de depressão em idosos atendidos em um ambulatório de gerontologia de Belo Horizonte. ConScientiae Saúde 2009;8(4):627-33.

21. Sousa M, Nunes A, Guimarães AI, Cabrita JM, Cavadas LF, Alves NF. Depressão em idosos: prevalência e fatores associados. Rev Port Clin Geral Fam 2010; 26(4):384-91.

22. Gomes JEM, Ruiz T, Corrente JE. Sintomas depressivos e déficit cognitivo na população de 60 anos e mais em um município de médio porte do interior paulista. Rev Bras Med Fam comunidade 2011;6(19):125-32.
23. Gazalle FK, De Lima MS, Tavares BF, Hallal PC. Sintomas depressivos e fatores associados em população idosa no Sul do Brasil. Rev Saúde Pública 2004;38(3):365-71.

24. Bós AMG, Bós AJG. Fatores determinantes e consequências econômicas da depressão entre os idosos no Brasil. Rev Bras Ciênc Envelh Hum 2005;2(2)36-46.

25. Batistoni SST, Neri AL, Cupertino APFB. Medidas prospectivas de sintomas depressivos entre idosos residentes na comunidade. Rev Saúde Pública 2010;44(6):1137-43.

26. Lebrão ML, Duarte YAO, organizadores. O Projeto Sabe no município de São Paulo: uma abordagem inicial. Brasília: OPAS. São Paulo; 2003.

27. Oliveira DAAP, Gomes L, Oliveira RF. Prevalência de depressão em idosos que frequentam centros de convivência. Rev Saúde Pública 2006;40(4):734-6.

28. Sousa RL, De Medeiros JGM, De Moura ACL, De Souza CLM, Moreira IF. Validade e fidedignidade da escala de depressão geriátrica na identificação de idosos deprimidos de um hospital geral. J Bras Psiquiatr 2007;56(2):102-7.

29. Snowdon J. Qual é a prevalência de depressão na terceira idade? Rev Bras Psiquiatr 2002;24(Supl 1):42-7.

30. Na YM, Kim KS, Lee KU, Chae JH, Kim JH, Kim DJ, et al. The Relationship between Depressive Symptoms in Outpatients with Chronic Illness and Health Care Costs. Yonsei Med J 2007;48(5):787-94.

31. Santo CAFE, Carneiro CFLC, De Lima HS, Alencar LBO, Pequeno OC. Determinantes do risco de depressão nos idosos em uma equipe da Estratégia de Saúde da Família [monografia de especialização]. Goiás: Universidade Federal de Goiás; 2010.

32. Prince MJ, Harwood RH, Thomas A, Mann AH. A prospective population-based cohort study of the effects of disablement and social milieu on the onset and maintenance of late-life depression. The Gospel Oak Project VII. Psychol Med 1998;28(2):337-50.

33. Santiago LM, Novaes CO. Auto-avaliação da audição em idosos. Rev CEFAC 2009;11(Supl 1):98-105. 